\title{
Feedback stabilisation of two-dimensional non-uniform pool-boiling states
}

\author{
R. W. van Gils, M. F. M. Speetjens \& H. Nijmeijer \\ Department of Mechanical Engineering, \\ Eindhoven University of Technology, The Netherlands.
}

\begin{abstract}
In this paper the feedback stabilisation of a two-dimensional pool-boiling system is discussed. The pool-boiling system is modelled by a so-called heater-only model, which consists of a Partial Differential Equation (PDE) which describes the heaters internal temperature field and its (nonlinear) boundary conditions given by the heat exchange between fluid and heater at the top and the heat supply at the bottom of the heater. The pool-boiling system is extended with an adjustable heat supply at the bottom of the heater, by which the unstable transition boiling regime is to be stabilised. Thereto, a feedback law, based on the Chebyshev-Fourier-cosine modes of the spectral discretisation of the temperature profile inside the heater, is applied to the system. The control parameters of this feedback law are designed such that satisfactory closed-loop dynamics are obtained. Using this specific feedback law, the control strategy is tested by simulations of the closed-loop pool-boiling system. Keywords: boiling, feedback, stabilisation, modal control, transition boiling, pool boiling, numerical simulation.
\end{abstract}

\section{Introduction}

Pool boiling may serve as physical model for cooling applications using boiling heat transfer. Boiling heat transfer is emerging as novel cooling technique, since it affords cooling capacities substantially beyond that of convential methods, which are based on air-cooling and single phase liquid cooling, see Mudawar [1], Khan et al [2] and Brooks et al [3]. Therefore, controlling the dynamical behaviour of pool boiling systems is the principal subject of investigations in this study.

A pool-boiling system is typically a system where a heater surface is submerged in a pool of saturated liquid. The heater surface is the fluid-heater interface where 
the actual boiling occurs. If the temperature of this heater surface is increased, three fundamental boiling modes can be observed, being nucleate, transition and film boiling. Nucleate boiling, at low temperatures, and film boiling, at high temperatures, are characterised by homogeneous liquid-rich and vapour-rich states of the fluid on the fluid-heater interface, respectively. They are the only stable modes in pool boiling. Furthermore, nucleate boiling is the most efficient boiling mode and, consequently, the desired state in cooling applications.

The transition nucleate-to-film boiling occurs if the heat generation exceeds the so-called "critical heat flux" (CHF) and thermal equilibrium is possible only in the film-boiling regime, see Dhir [4]. The intermediate regime is characterised by the formation and rapid evolution of highly-unstable heterogeneous boiling states (i.e. coexisting liquid-rich and vapour-rich regions) on the fluid-heater interface. Due to the jump in system temperature, the transition is accompanied by collapse of cooling capacity. Hence, optimal cooling performance is a trade-off between close proximity to the CHF (efficient nucleate boiling) and a safety margin (prevention of transition), see Mudawar [1] and Chu et al [5].

Aim of the present study is the model-based development of a control strategy that allows boiling close to the CHF. This control strategy is to be developed by means of the compact pool boiling model first introduced in Speetjens et al [6]. This model describes the pool boiling dynamics entirely in terms of the temperature field within the heater. The system thus reduces to a PDE which describes the heater's internal temperature field and its (nonlinear) boundary conditions, i.e. adiabatic sidewalls, the heat exchange between fluid and heater at the top and the heat supply at the bottom of the heater.

In van Gils et al [7] the stabilisation of the transition boiling regime is investigated for a one-dimensional (1D) simplification of the heater-only model. A uniform transition state is stabilised by means of a simple linear feedback law based on the dominant modes of the spatial discretisation of the temperature profile in the heater. Analyses of this so-called modal controller, propose it as a viable option for the rapid stabilisation and regulation of the pool-boiling system.

In this study, the objective is stabilisation of the two-dimensional (2D) transition boiling states. Thereto, the control strategy employed in van Gils et al [7] is applied to the $2 \mathrm{D}$ system. The effectiveness of this method is checked by testing a specific feedback law, found to stabilise the linearised system, using simulations of the closed-loop nonlinear system.

This paper is organised as follows. In Section 2 a short introduction to the model and its steady states is given. Then in Section 3 the used control strategy and the method to analyse the unstable equilibria of this model are introduced. Subsequently, Section 4 treats the simulations of the system where an unstable equilibrium is stabilised by the proposed controller. Finally, in Section 5 some conclusions are drawn. 


\section{Pool-boiling model description}

Pool-boiling systems are represented in this study by a heater-only model first presented in Speetjens et al [6]. In this model the boiling fluid is solely modelled by the boundary condition on top of the heater. This is allowed because of a phenomenology connection between the boiling mode and the interface temperature, cf. Speetjens et al [6].

\subsection{Heater-only model}

The heat transfer within the $2 \mathrm{D}$ rectangular heater $\mathcal{H}:=[0,1] \times[0, D]$, see Figure 1 (a) is considered. Its boundary conditions are given by (i) adiabatic sidewalls for $x=0,1$, (ii) a constant heat supply extended with the system input, by which unstable states must be stabilised at $y=0$, and (iii) the nonlinear heat extraction by the boiling process at $y=D$. The heat transfer within $\mathcal{H}$ is modelled by

$$
\frac{\partial T}{\partial t}=\kappa \nabla^{2} T
$$

where the field $T=T(x, y, t)$ is the non-dimensional temperature excess. The boundary conditions to this PDE are given by

$$
\left.\frac{\partial T}{\partial x}\right|_{x=0,1}=0,\left.\quad \frac{\partial T}{\partial y}\right|_{y=0}=-\frac{1}{\Lambda}(1+u(t)),\left.\quad \frac{\partial T}{\partial y}\right|_{y=D}=-\frac{\Pi_{2}}{\Lambda} q_{F}\left(T_{F}\right)
$$

where $T_{F}:=T(x, D, t)$ is the temperature at the fluid heater interface, $\Lambda$ and $\kappa$ the nondimensional heater conductivity and diffusivity, respectively, $D$ the heater aspect ratio and $\Pi_{2}$ the ratio between CHF and constant heat supply. Since physical considerations imply $\Lambda D / \kappa=\left|1-\Pi_{2}\right|, \Lambda, D$ (heater properties) and $\Pi_{2}$ (heating conditions) are left as remaining system parameters. In this study these system parameters are given by $\Lambda=D=0.2$ and $\Pi_{2}=2$. Furthermore, the nonlinear heat-flux function $q_{F}\left(T_{F}\right)$ describes the local heat exchange between the heater and the boiling fluid. On physical grounds, it is identified with the so-called boiling curve, that is, the relation describing the mean heat exchange between heater and fluid along the entire fluid-heater interface. This implies a functional relation $q_{F}\left(T_{F}\right)$ according to Figure 1(b). Here $T_{F}<1$ and $T_{F}>1$ corresponds to local nucleate and local film boiling regions, respectively, see Speetjens et al [6].

\subsection{Equilibria of the model}

An extensive exposition on the steady states of this model and their stability properties is furnished in Speetjens et al $[6,8]$. Below a concise recapitulation is provided. 


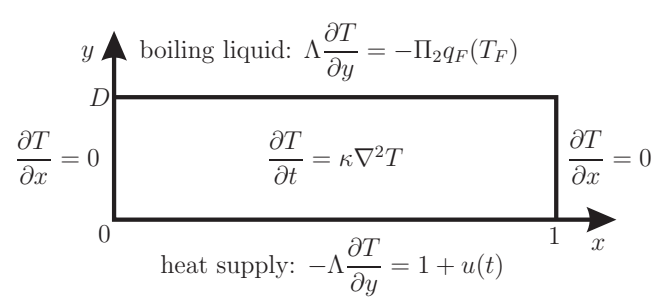

(a) Heater configuration

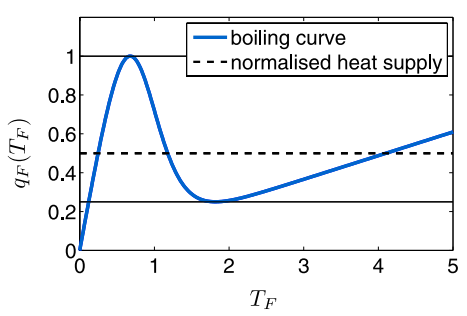

(b) Heat flux function

Figure 1: Non-dimensional model: heater configuration (a) and heat-flux function (heavy line) and normalised heat supply (dashed line) (b).

Steady states $T_{\infty}(x, y)$ of eqn. (1) are found via application of the method of separation of variables. This yields a (formal) solution given by

$$
T_{\infty}(x, y)=\sum_{n=0}^{\infty} \widetilde{T}_{n} \frac{\cosh (n \pi y)}{\cosh (n \pi D)} \cos (n \pi x)+\frac{D-y}{\Lambda}
$$

with coefficients $\widetilde{T}_{n}$ the spectrum of the Fourier cosine expansion

$$
T_{F, \infty}(x):=T_{\infty}(x, D)=\sum_{n=0}^{\infty} \widetilde{T}_{n} \cos (n \pi x)
$$

of the temperature at the fluid-heater interface. These coefficients are determined by the nonlinear Neumann condition at $y=D$, upon substitution of eqn. (3) leading to

$$
\sum_{n=0}^{\infty} n \pi \tanh (n \pi D) \widetilde{T}_{n} \cos (n \pi x)+\frac{\Pi_{2}}{\Lambda} q_{F}\left(T_{F, \infty}(x)\right)-\frac{1}{\Lambda}=0
$$

for all $x \in[0,1]$. Eqn. (5) is the characteristic equation that determines the particular properties of the steady states of eqn. (1). If $\widetilde{T}_{n}=0$ for $n>0$, the equilibrium is constant in $x$-direction and eqn. (5) simplifies to $q_{F}\left(T_{F, \infty}\right)=\Pi_{2}^{-1}$. As a result, $T_{F, \infty}$ coincides with the intersection(s) between the boiling curve (solid line Figure 1(b)) and the normalised heat-supply (dashed line in Figure 1(b)). Here the left and right intersections correspond to stable nucleate and stable film boiling, respectively. The middle intersection corresponds to an unstable transition boiling equilibrium. These three equilibria are called the homogeneous equilibria as they are uniform in $x$-direction. All other equilibria are not uniform in $x$ direction and are called the heterogeneous equilibria. Analyses reveal that a conjugate steady state solution, given by $T_{\infty}^{*}(x, y)$, exists for each heterogeneous equilibrium. The conjugate solution of the heterogeneous equilibrium considered in 


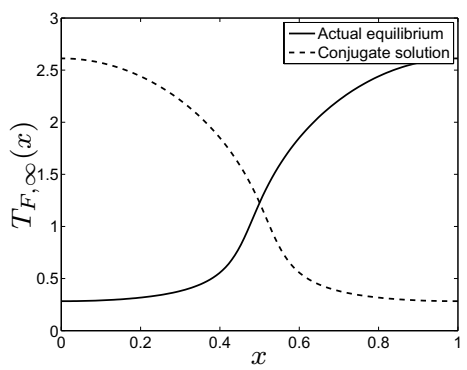

Figure 2: Fluid heater interface temperature of the heterogeneous equilibrium and its conjugate solution.

this study exhibit the following symmetry property

$$
T_{\infty}^{*}(x, y)=T_{\infty}(x+1, y), \quad x \in[0,1], y \in[0, D] .
$$

The temperature distribution on the fluid-heater interface of the heterogeneous equilibrium and its conjugate solution considered in this study are given in Figure 2. Heterogeneous solutions are specified by regions which are locally rich in liquid, $T_{F}<1$, or vapour, $T_{F}>1$ and thus belong to the transition boiling regime. Furthermore, these equilibria are highly unstable and evolve rapidly towards one of the stable homogeneous equilibria, see Speetjens et al [8]. This complicates investigation of these states substantially.

Principal objective of this study is the stabilisation of these inherently unstable equilibria. Since their conjugate solutions govern exactly the same properties, stabilisation of a heterogeneous equilibrium automatically means stabilisation of its conjugate solution and vice versa. Hence, only the stabilisation of the actual solution is discussed here.

\subsection{Linearisation around a specific equilibrium}

To determine the stability properties (open-loop and closed-loop) of an equilibrium considered, the nonlinear system is linearised around it. Small perturbations $v(x, y, t)$ around the equilibrium $T_{\infty}(x, y)$, are considered, i.e. $T(x, y, t)=$ $T_{\infty}(x, y)+v(x, y, t)$. The linearised system then is given by

$$
\frac{\partial v}{\partial t}=\kappa \nabla^{2} v
$$

with the boundary conditions

$$
\left.\frac{\partial v}{\partial x}\right|_{x=0,1}=0,\left.\quad \frac{\partial v}{\partial y}\right|_{y=0}=-\frac{1}{\Lambda} u(t),\left.\quad \frac{\partial v}{\partial y}\right|_{y=D}=-\frac{\Pi_{2}}{\Lambda} \gamma(x) v(x, D, t),
$$

where $\gamma(x)=\frac{\partial q_{F}\left(T_{F}(x)\right)}{\partial T_{F}}$, see Speetjens et al [8]. 


\section{Control strategy}

In this section the control strategy for stabilisation of the pool boiling system is discussed. The intended control strategy is a feedback law, based on the deviation of the internal temperature excess of the heater from its equilibrium, i.e. $v(x, y, t)$. Furthermore, the aim is to control the pool-boiling system using an uniform heat supply. Hence, it must be a scalar. This results in the following feedback law

$$
u(t)=\int_{0}^{D} \int_{0}^{1} v(x, y, t) g(x, y) \mathrm{d} x \mathrm{~d} y
$$

where $g(x, y)$ is a feedback weight function with which the properties of the feedback law will be prescribed. Furthermore, the temperature inside the heater $v(x, y, t)$ is expressed in a form that is intimately related to its natural eigenmodes, which are the eigenmodes of the Laplace operator. This means that in $x$-direction, the eigenmodes of the Laplace operator are taken to represent the profile. In $x$ direction the profile thus is represented by a Fourier-cosine expansion. Hence, the boundary conditions on $x=0,1$ are automatically fulfilled by this expansion. In $y$-direction the profile is expressed in the non-periodic variant of this expansion, i.e. the Chebyshev expansion. The temperature profile is thus given by

$$
v(x, y, t)=\sum_{k=0}^{\infty} \sum_{n=0}^{\infty} \widetilde{v}_{n k}(t) \phi_{n}(\theta(y)) \cos (k \pi x)
$$

where $\phi_{n}(\theta)=\cos (n \arccos (\theta))$ is the $n^{\text {th }}$ Chebyshev polynomial and $\theta=$ $\frac{2}{D} y-1$ is the computational domain, cf. Canuto et al [9]. Note that here an infinite series is considered, meaning no approximation error is introduced in this step. Furthermore, if a smooth temperature field in the heater is assumed, due to exponential convergence of the Chebyshev-Fourier-cosine spectral coefficients, the dynamics of the system are mainly prescribed by the 'lower order' modes, i.e. modes with low $n$ and low $k$. As a result, the feedback law given by eqn. (9) only needs to be based on the 'lower' modes, as established in van Gils et al [7].

In order to filter these specific modes from the profile $v(x, y, t)$, the feedback weight function $g(x, y)$ is taken as

$$
g(x, y)=\sum_{q=0}^{\infty} \sum_{p=0}^{\infty} \widetilde{g}_{q p} \cos (q \arccos (\theta(y))) w_{C}(\theta(y)) \cos (p \pi x) w_{F}(x)
$$

where $w_{C}(\theta)=\left(1-\theta^{2}\right)^{-\frac{1}{2}}$ the orthogonal weight function of the Chebyshev polynomials, $w_{F}(x)=1$ the orthogonal weight function of the Fourier-cosine polynomials, cf. Canuto et al [9] and $\widetilde{g}_{q p}$ the spectral coefficients of the weight 
function. Due to the orthogonality property of the Chebyshev and Fourier polynomials, implementing eqn. (10) and eqn. (11) in eqn. (9) reduces the feedback law to

$$
u(t)=\frac{D}{2} \sum_{q=0}^{\infty} \sum_{p=0}^{\infty} \widetilde{v}_{q p}(t) \widetilde{g}_{q p} \bar{C}_{q} \widetilde{C}_{p}=\sum_{q=0}^{\infty} \sum_{p=0}^{\infty} \widetilde{v}_{q p}(t) k_{q p},
$$

where $k_{q p}=\frac{D}{2} \bar{C}_{q} \widetilde{C}_{p} \widetilde{g}_{q p}$ and the factors $\bar{C}_{i}=\int_{-1}^{1} \phi_{n}^{2}(\theta) w_{C}(\theta) \mathrm{d} \theta$ and $\widetilde{C}_{i}=$ $\int_{0}^{1} \cos ^{2}(k \pi x) w_{F}(x) \mathrm{d} x$ are given in Canuto et al [9]. Hence, this enables control of individual Chebyshev-Fourier-cosine modes by appropriate choice of $k_{q p}$. This socalled modal control scheme thus enables efficient control of exactly the relevant lower order modes.

The elements $k_{q p}$ must be determined such that the closed-loop poles lie at desired/satisfactory locations. To determine these closed-loop poles, a characteristic equation is derived analogously to the derivation of eqn. (5). The closed-loop poles of the system are given by the $\lambda \in \mathbb{C}$ that satisfy

$$
\begin{aligned}
& \sum_{k=0}^{\infty} A_{k} \cos (k \pi x)\left[\sqrt{\alpha_{k}} \sinh \left(\sqrt{\alpha_{k}} D\right)+\frac{\Pi_{2}}{\Lambda} \gamma(x) \cosh \left(\sqrt{\alpha_{k}} D\right)\right]+ \\
& {\left[\frac{\Pi_{2}}{\Lambda} \gamma(x)-\sqrt{\alpha_{0}}\right] \frac{\frac{1}{\Lambda} \sum_{k=0}^{\infty} \sum_{q=0}^{\infty} k_{q k} \xi_{q}^{k} A_{k}}{\sqrt{\alpha_{0}}-\frac{1}{\Lambda} \sum_{q=0}^{\infty} k_{q 0} \zeta_{q}^{-}} e^{-\sqrt{\alpha_{0}} D}=0, \quad \forall x \in[0,1], }
\end{aligned}
$$

for nontrivial $A_{k}$ (i.e. $A_{k} \neq 0 \forall k$ ). The parameter $\alpha_{k}$ is given by

$$
\alpha_{k}=(k \pi)^{2}+\frac{\lambda}{\kappa} .
$$

Furthermore, $\left(A_{0} \cdots A_{\infty}\right)$ is the spectrum of the eigenmode of the closed-loop system that corresponds to the computed pole $\lambda$. The coefficients $\xi^{k}$ and $\zeta^{-}$are the spectral coefficients of the Chebyshev expansion of $\cosh \left(\sqrt{\alpha_{k}} \frac{D}{2}(\theta+1)\right)$ and $e^{\sqrt{\alpha_{0}} \frac{D}{2}(\theta+1)}$, respectively. Approximations to the poles of the closed-loop system can be found via discretisation of eqn. (13) by the method according to Speetjens et al [8].

Using this equation the closed-loop poles for a specific feedback law, i.e. with the elements $k_{n k}$ given, can be determined. By varying one of the elements of the feedback law, e.g. $k_{0,0}$, the poles will move away from their original position. By plotting the path the dominant poles describe as function of the controller element, a pole-trajectory plot can be obtained. Note, that the dominant poles in the openloop case not necessarily are dominant poles in the closed-loop case. Therefore, it must be monitored whether there are poles that move towards the right half plane and in that way become dominant closed-loop poles. Using the pole-trajectory plots, controllers that result in satisfying closed-loop behaviour are designed. 


\section{Simulations}

As is mentioned in the previous section, the system linearised around the heterogeneous equilibrium will be stabilised by means of a linear state feedback based on the dominant modes of the temperature profile. Important to note here is that these spatially varying equilibria are stabilised using a spatially constant input.

Whether the controller, designed using the linearised system, can handle the nonlinearity of the pool boiling model is examined in this section. Since the linearised system is stabilised, the nonlinear system should at least be stabilised for small deviations around the equilibrium. However, the size of the so-called region of attraction, in which initial conditions still evolve asymptotically to the equilibrium, is not known a priori. In the following it is shown that the initial offsets during simulations can be taken quite large, implying a relatively large region of attraction.

In this section the evolution of the fluid-heater interface temperature during simulations of the closed-loop pool-boiling system is given. For the numerical simulations, the spatial discretisation of eqn. (1) and eqn. (2) is used. The discretisation is performed by a spectral method based on the Chebyshev-Fourier-cosine expansion, given in eqn. (10). The boundary conditions on $y=0$ and $y=D$ are implemented by the tau-method. Finally, the time discretisation is done using a second-order Cranck-Nicholson time-marching scheme, see Canuto et al [9] and van Gils et al [7]. Each Fourier-mode is solved individually. However, as a result of the spatial dependence of the nonlinear Neumann boundary condition on top of the heater, the Fourier modes are coupled by this boundary condition. This spatial dependence is treated by the Picard iteration scheme, Kreyszig [10].

Simulations are done for systems which have an initial offset from the to-bestabilised equilibrium. The initial profiles are generated in a similar way as is done in van Gils et al [7]. This means a perturbed Chebyshev-Fourier-cosine spectrum of one of the systems equilibria, serves as initial condition for the simulations. The perturbation consists of a super imposed offset $\Delta T=\epsilon \mathbf{n}$, with $\mathbf{n}$ an exponentiallydecaying Gaussian noise vector with mean zero and covariance 0.5 , so as to obtain a smooth initial temperature profile. The coefficient $\epsilon$ determines the magnitude of the initial deviation.

\subsection{Stabilisation of the heterogeneous equilibrium}

The methodology described in the previous section is applied to design stabilising controllers for the heterogeneous equilibrium, shown in Figure 2. Modal controllers that regulate only the zero-th Chebyshev-Fourier-cosine mode, i.e. $\widetilde{g}_{0,0} \neq 0$ and the first Chebyshev, zero-th Fourier-cosine mode, i.e. $\widetilde{g}_{1,0} \neq 0$ are considered. Hence, $\widetilde{g}_{0,0} \neq 0, \widetilde{g}_{1,0} \neq 0$ and all other controller elements equal zero. Satisfactory gain values are found via pole trajectory plots. The values

$$
k_{0,0}=-2, \quad k_{1,0}=-3
$$




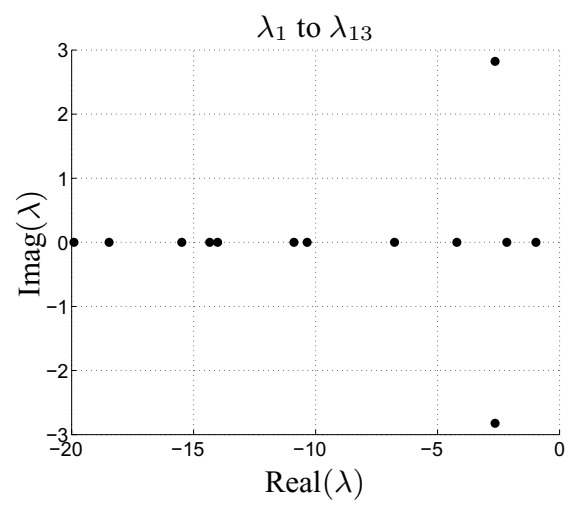

Figure 3: Dominant poles of the closed-loop system.

are used in these simulations, since for these parameters the dominant closed-loop poles lie furthest away from the imaginary axis (if only this $\widetilde{g}_{0,0}$ and $\widetilde{g}_{1,0}$ are taken nonzero). The dominant closed-loop poles of the linearised system are given in Figure 3.

In Figure 4(a) and (b) the simulation of the nonlinear and linear system are shown, respectively. The initial condition in these simulations have a deviation from the heterogeneous equilibria of $\epsilon=10^{-0.7}$. The evolution of the fluid-heater interface temperature is shown. The horizontal lines represent the three homogeneous equilibria. Furthermore, the dashed non-uniform line is the heterogeneous equilibrium and the dash-dotted line is its conjugate state. As can be seen, although the initial deviations from the equilibrium are quite large, the nonlinear system still is stabilised by the linear controller. Here in both simulations the state evolves to the heterogeneous equilibrium. Whether the system evolves to the actual solution or its conjugate solution, can not be influenced by the controller, it depends solely on the initial temperature profile of the heater as will be shown in the following. Important to notice is that this non-uniform equilibrium is stabilised by a uniform adjustable heat supply at the bottom of the heater.

In Figure 4(c) the mean interface temperature of the linear and nonlinear system is given as function of time. As can be seen the mean temperature oscillates to its setpoint. This is the result of the complex poles shown in Figure 3. This is observed in Figure 4(d) as well, where the input is given as function of time for both the linear and nonlinear system.

Simulations with the stable film boiling equilibrium as initial condition are also performed. Here a deviation of magnitude $\epsilon=10^{-3}$ is added. The evolution plot for the nonlinear and the linear simulation is shown in Figure 5(a) and (b), respectively. As can be seen the nonlinear system converges to the conjugate state. This is due to the initial deviation between the state and the equilibrium. The initial fluctuations are amplified by the nonlinear boundary condition on the top of the heater. If the mean error between the initial profile and the homogeneous equilibrium is 


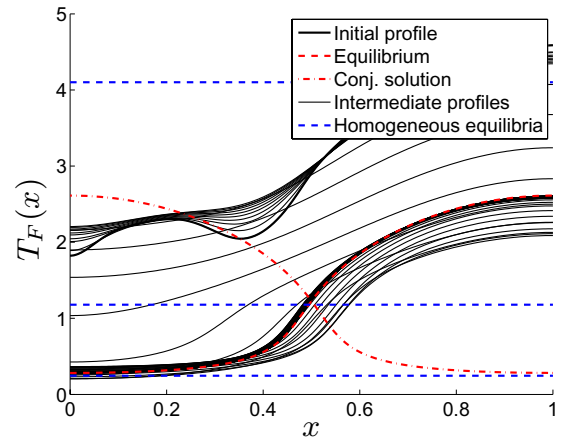

(a) Nonlinear system

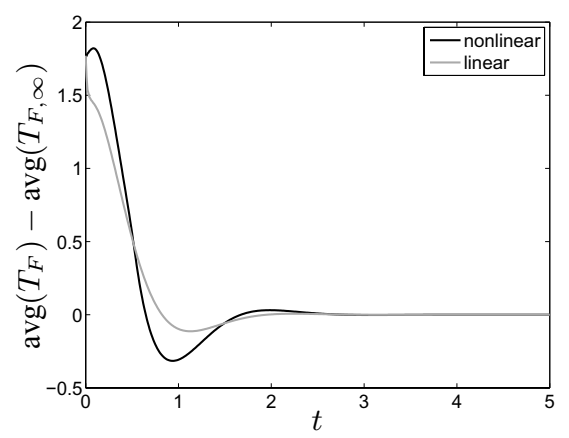

(c) Mean fluid heater interface as function of time

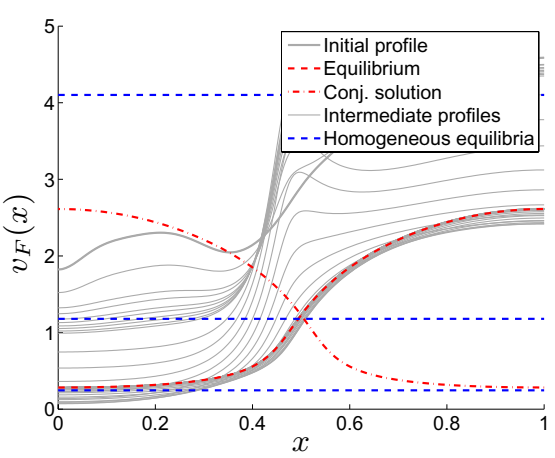

(b) Linear system

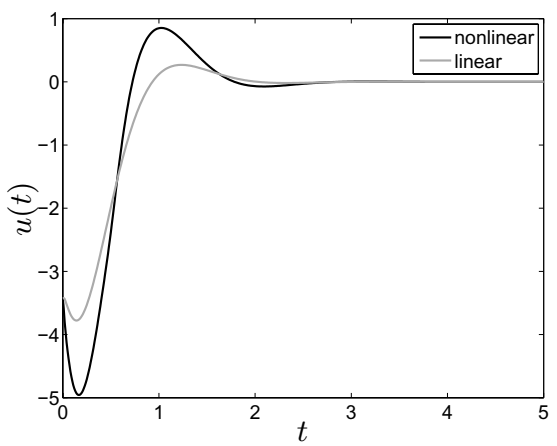

(d) Input as function of time

Figure 4: Nonlinear and linear results. Evolution of fluid-heater interface temperature of the nonlinear (a) and linear (b) system. Mean fluid-heater interface temperature as function of time (c) and the input as function of time (d).

larger for $x \in[0,0.5]$ than for $x \in[0.5,1]$, the temperature profile will evolve to the conjugate equilibrium. Vice versa, the temperature profile will evolve to the actual equilibrium.

In the simulation of the linear system, the fluid-heater interface temperature does not evolve to the conjugate equilibrium, since the heterogeneous equilibria are solutions of the nonlinear system and their conjugate solution does not exist in the linear system (linear systems always have only one equilibrium). This thus is an example where the linear system can not describe the nonlinear system anymore. Nevertheless, the linear controller manages to stabilise the heterogeneous equilibrium. Conversely, analysis of the linearised system yields the controller with elements as in eqn. (15) by which the nonlinear system can be stabilised. Moreover, the applied heat supply at the bottom of the heater is uniform in $x$ and $y$ direction. The heterogeneity thus is solely the result of the nonlinear Neumann boundary condition at the fluid-heater interface. 


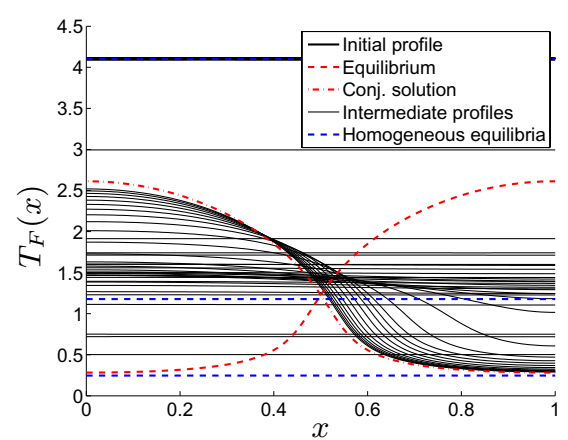

(a) Nonlinear system

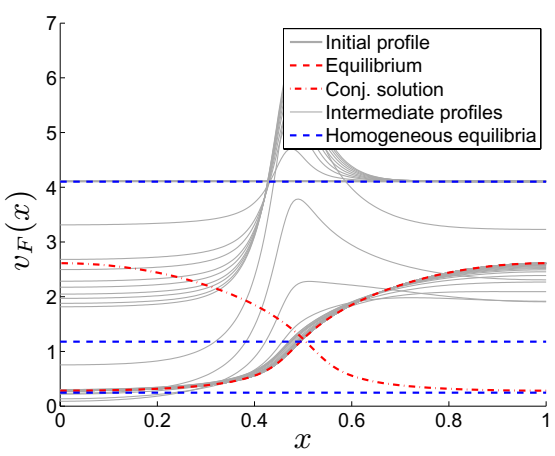

(b) Linear system

Figure 5: Evolution of fluid-heater interface temperature.

\section{Conclusions}

In this study a 2D nonlinear heat-transfer model for pool-boiling systems is considered. The model involves only the temperature distribution within the heater and models the heat exchange with the boiling medium via a nonlinear boundary condition imposed at the fluid-heater interface. This compact model is employed for the design and analysis of a robust control strategy for the stabilisation of unstable transition boiling states. To this end a controller is introduced that regulates the heat supply as a function of the internal state. Previous analysis have shown that controllers based on the dominant modes of the spatial discretisation of the temperature profile within the heater are effective, see van Gils et al [7]. For this reason the controller developed to be applied to this system is based on these modes as well.

The control strategy filters the Chebyshev-Fourier-cosine modes of the temperature profile. Due to the structure of the heater-only model, these modes are intimately related to the eigenmodes of the system. Therefore, the dynamics are mainly prescribed by the dominant modes, that is, the lower order ChebyshevFourier-cosine modes. The feedback law thus enables control of exactly those relevant lower order modes.

Satisfactory closed-loop behaviour is obtained by fine-tuning of the control parameters. This is done by means of pole trajectory plots. These plots show the path the closed-loop poles describe as function of a control parameter. Closed-loop poles are found by solving a characteristic equation found by direct analysis of the heater-only model, using the method of separation of variables.

The performance of the control law for stabilisation of the nonlinear system is investigated in order to establish its value for practical purposes. Relevant issues are the asymptotic stability and evolution of the nonlinear closed-loop system. Simulations of the evolution of the nonlinear system reveal convergence on the unstable steady state for a wide range of initial states. These findings imply that 
the control loop indeed is capable of robustly stabilising the pool-boiling system. Especially the fact that non-uniform equilibria are stabilised here by means of an uniform input must be emphasised. Even when the initial condition is almost uniform in $x$-direction, the feedback law manages to bring the system to the nonuniform solution. Here the heterogeneity is solely introduced by the nonlinear Neumann condition on the fluid-heater interface.

\section{References}

[1] Mudawar, I., Assessment of high-heat-flux thermal management schemes. IEEE Transactions on Components and Packaging Technologies, 24(2), pp. 122-141, 2001.

[2] Khan, N., Pinjala, D. \& Toh, K., Pool boiling heat transfer enhancement by surface modification/micro structures for electronics cooling: a review. Electronic Packaging Technology Conference, 2004.

[3] Brooks, D. \& Martonosi, M., Dynamic thermal management for highperformance microprocessors. Seventh International Symposium on HighPerformance Computer Architecture (HPCA-7), 2001.

[4] Dhir, V., Boiling heat transfer. Annu Rev Fluid Mech, 30(1), pp. 365-401, 1998.

[5] Chu, R., Simons, R., Ellsworth, M., Schmidt, R. \& Cozzolino, V., Review of cooling technologies for computer products. IEEE Transactions on Device and Materials Reliability, 4(4), pp. 568-585, 2004.

[6] Speetjens, M., Reusken, A. \& Marquardt, W., Steady-state solutions in a nonlinear pool boilingmodel. Communications in Nonlinear Science and Numerical Simulation, 13(8), pp. 1475-1494, 2008.

[7] van Gils, R., Speetjens, M. \& Nijmeijer, H., Feedback stabilisation of a onedimensional nonlinear pool-boiling system. International Journal of Heat and Mass Transfer, 53(11-12), pp. 2393-2403, 2010.

[8] Speetjens,M., Reusken, A.,Maier-Paape, S. \& Marquardt,W., Stability analysis of two-dimensional pool-boiling systems. SIAM J Appl Dyn Syst, 7(3), pp. 933-961, 2008.

[9] Canuto, C., Hussaini, M., Quarteroni, A. \& Zang, T., Spectral Methods in Fluid Dynamics. Springer, New York, 3rd edition, 1987. Ch. 2.

[10] Kreyszig, E., Advanced Engineering Mathematics. Wiley, New York, eighth edition, 1999. Ch. 11. 